\title{
Study Protocol for Pleiotropic Effects and Safety of Sodium-Glucose Cotransporter 2 Inhibitor Versus Sulfonylurea in Patients with Type 2 Diabetes and Nonalcoholic Fatty Liver Disease
}

\author{
Yumie Takeshita - Takehiro Kanamori - Takeo Tanaka - Yuka Kaikoi • Yuki Kita • \\ Noboru Takata · Noriho Iida - Kuniaki Arai - Tatsuya Yamashita - Kenichi Harada • \\ Toshifumi Gabata $\cdot$ Hiroyuki Nakamura $\cdot$ Shuichi Kaneko $\cdot$ Toshinari Takamura (D)
}

Received: November 26, 2019 / Published online: January 20, 2020

(c) The Author(s) 2020

\begin{abstract}
Introduction: Clinicopathological analyses revealed that reduction in $\mathrm{HbA1c}$ and use of insulin independently contribute to reduction in liver fibrosis scores during the course of nonalcoholic fatty liver disease (NAFLD) development. We will test our hypothesis that lowering glucose and increasing insulin reduce liver fibrosis in NAFLD. Sodium-glucose cotransporter 2 (SGLT2) inhibitors lower insulin levels and sulfonylureas increase insulin levels, while both lower glucose levels.

Methods: This study is a 48-week, one-center (only Kanazawa University Hospital), open-label, randomized, parallel trial. Patients who
\end{abstract}

Enhanced Digital Features To view enhanced digital features for this article go to https://doi.org/10.6084/ m9.figshare.11534343.

Y. Takeshita · T. Kanamori · T. Tanaka · Y. Kaikoi ·

Y. Kita · T. Takamura $(\square)$

Department of Endocrinology and Metabolism, Kanazawa University Graduate School of Medical Sciences, 13-1 Takara-machi, Kanazawa, Ishikawa 920-8640, Japan

e-mail: ttakamura@med.kanazawa-u.ac.jp

N. Takata $\cdot$ N. Iida $\cdot$ K. Arai · T. Yamashita .

S. Kaneko

Department of Gastroenterology, Kanazawa

University Graduate School of Medical Sciences, 13-

1 Takara-machi, Kanazawa, Ishikawa 920-8640,

Japan satisfied the eligibility criteria were randomly assigned (1:1) to receive once-daily $20 \mathrm{mg}$ tofogliflozin or $0.5 \mathrm{mg}$ glimepiride. The sample size was calculated to be 14 in each group with a significance level of 0.05 and power of 0.90 . The design required 40 evaluable patients in this study. The primary endpoint of this study will be the improvement in liver histology between liver biopsies at baseline and after 48 weeks of treatment. The secondary efficacy endpoints in the present study include organ-specific insulin sensitivity, insulin/glucagon secretion, ectopic fat accumulation, bioelectrical impedance analysis, sympathetic nerve activity, comprehensive gene expression analyses in the liver and blood cells, and gut microbiota profiling.

Planned Outcomes: Recruitment into this study started in November 2015 and will end in

\footnotetext{
K. Harada

Department of Human Pathology, Kanazawa University Graduate School of Medicine, Kanazawa 920-8640, Japan

T. Gabata

Department of Radiology, Kanazawa University Graduate School of Medicine, 13-1 Takara-machi, Kanazawa 920-8640, Japan

H. Nakamura

Department of Environmental and Preventive Medicine, Faculty of Medicine, Institute of Medical, Pharmaceutical and Health Sciences, Kanazawa University, 13-1 Takara-machi, Kanazawa 920-8640, Japan
} 
September 2020, with 40 patients randomized into the two groups. The treatment follow-up of the participants is currently ongoing and is due to finish by the end of 2022 . The findings of this trial will be disseminated through peer-reviewed publications and international presentations.

Trial Registration: This trial is registered with the University Hospital Medical Information Network Clinical Trials Registry (UMIN0000 20544) and ClinicalTrials.gov (NCT02649465).

Keywords: Nonalcoholic fatty liver disease; Sodium-glucose cotransporter 2 inhibitor; Sulfonylurea; Treatment strategies; Type 2 diabetes

\section{Key Summary Points}

Clinicopathological analyses led us to hypothesize that glycemic control and insulin therapy ameliorate or protect against the histological progression of liver fibrosis in patients with nonalcoholic steatohepatitis. To test this hypothesis, we aim to compare the effects of Sodiumglucose cotransporter 2 (SGLT2) inhibitors and sulfonylureas, which lower glucose levels by decreasing and increasing circulating levels of insulin, respectively, in patients with type 2 diabetes.

Previous clinical trials lacked a control group or histological examination, which precludes meaningful conclusions since the natural course of the disease or tight glycemic control may ameliorate liver pathology in some patients with nonalcoholic fatty liver disease (NAFLD). We investigated the efficacy of the SGLT2 inhibitor tofogliflozin and the sulfonylurea glimepiride in liver pathology in patients with NAFLD and T2D for 48 weeks in an open-label, randomized, parallel study.
Using many metabolic markers (hyperinsulinemic euglycemic clamp study, arginine stimulation test, ${ }^{1} \mathrm{H}$ MRS (magnetic resonance spectroscopy), bioelectrical impedance analysis, Holter electrocardiograms, liver and blood cells, and gut microbiota profiling), we may be able to clarify the mechanisms underlying the SGLT2 inhibitor/sulfonylureamediated alteration in body weight and whole-body energy metabolism.

\section{INTRODUCTION}

Nonalcoholic fatty liver disease (NAFLD), ranging from simple fatty liver to nonalcoholic steatohepatitis (NASH), is a liver phenotype of metabolic disorders, such as diabetes, obesity, and metabolic syndrome. Hepatic steatosis may be a cause and consequence of insulin resistance. However, to date, how steatosis is linked to inflammation and fibrosis in NASH remains to be elucidated. Hepatic fibrosis is associated with liver cirrhosis and hepatocellular carcinoma, which determine the prognosis in patients with NASH. We previously investigated the histological course of serial liver biopsy samples of patients with NAFLD in a real-world clinical setting. The clinicopathological analyses revealed that reduction in HbA1c and use of insulin independently contribute to reduction in liver fibrosis scores during the course of NAFLD development [1]. These findings led us to hypothesize that glycemic control and insulin therapy ameliorate or protect against the histological progression of liver fibrosis in patients with NASH.

To test this hypothesis, we aim to compare the effects of sodium-glucose cotransporter 2 (SGLT2) inhibitors and sulfonylureas, which lower glucose levels with decreases and increases in circulating levels of insulin, respectively, in patients with type 2 diabetes. Both of these 
antidiabetic agents are chosen as second-line therapy when glycemic control cannot be achieved with metformin or as first-line therapy when metformin is contraindicated or not tolerated [2]. The SGLT2 inhibitors reduce weight and body fat mass [3], whereas sulfonylureas may increase them. In animal models of NAFLD/NASH, SGLT2 inhibitors protect against steatosis, inflammation, and fibrosis $[4,5]$. The protective effects have been considered as a consequence of glycosuria-induced negative energy balance and substrate switching toward lipids as a source of energy expenditure [6]. Previous clinical trials have demonstrated that SGLT2 inhibitors exert protective effects on liver enzymes and liver steatosis in patients with NAFLD/NASH [7, 8]. However, these studies lack a control group or histological examination, which precludes meaningful conclusions, since the natural course of the disease or tight glycemic control may ameliorate liver pathology in some patients with NAFLD [1]. Sulfonylureas are still reliable and strong antidiabetic agents in insulinopenic patients with type 2 diabetes and are, therefore, used as second-line therapies, especially when the cost is a major issue. Sulfonylureas have the advantage of lowering glucose, but they increase weight [9], which may render positive and negative effects on liver pathology in NAFLD/NASH.

In the present study, we are investigating the efficacy of the SGLT2 inhibitor tofogliflozin and sulfonylurea glimepiride in liver pathology in patients with NAFLD and type 2 diabetes for 48 weeks in an open-label, randomized, parallel study.

\section{METHODS}

\section{Study Design}

This study is a 48-week, one-center (only Kanazawa University Hospital), open-label, randomized, parallel trial of treatment with $20 \mathrm{mg}$ tofogliflozin or $0.5 \mathrm{mg}$ glimepiride for adults with type 2 diabetes and biopsy-proven NAFLD.

The study was conducted with the approval of the Certified Review Board, Kanazawa
University, Ishikawa, Japan, in accordance with the guidelines of the Declaration of Helsinki (2018-2020, jRCTs 041180132). Written informed consent was obtained from all of the subjects before enrollment in the study. This trial is registered with the University Hospital Medical Information Network Clinical Trials Registry (UMIN000020544) and ClinicalTrials.gov (NCT02649465).

\section{Sample Selection}

\section{Eligibility for the Trial}

Eligible adults (at least 20 years old) were identified and recruited at Kanazawa University Hospital from November 2015 until September 2020. All of the trial participants provided their informed written consent at the beginning of the screening visit before this study.

Eligibility for the trial was determined at screening visit 1 using standard blood tests, clinical history (including written confirmation of drug history, where necessary), and physical examination/observations to identify other illnesses or contraindications. The patients who satisfied the eligibility criteria for the 48-week treatment trial at Kanazawa University Hospital were given the option to participate in metabolic mechanistic sub-studies, including the hyperinsulinemic euglycemic clamp study with stable isotope-labeled glucose infusion and arginine stimulation tests at visit 2 . A patient's decision to partake or withdraw from the metabolic sub-study did not affect their participation in the main 48-week trial.

Fatty liver is clinically diagnosed on the basis of ultrasound examinations showing an increase in hepatorenal contrast. We defined hepatorenal contrast, also known as "bright liver", as a ratio of hepatic to kidney echo levels of greater than 1.0. We excluded all other liver disorders in each patient. All patients reported drinking less than $20 \mathrm{~g}$ /day of ethanol. All liver biopsies were performed during hospitalizations. All biopsies were obtained after a thorough clinical evaluation and obtaining receipt of signed informed consent from each patient.

The trial entry criteria are based on a diagnosis of "definite" NAFLD with type 2 diabetes 
on liver biopsy obtained within 3 months of screening. All of the participants had to be at least 20 years of age at the time of the initial screening. Patients with type 2 diabetes mellitus at the time of screening need to have glycemic control (HbA1c of at least 7\%) and have been managed by either diet and/or a stable dose of hypoglycemic agents for at least 4 weeks.

The exclusion criteria included hepatic virus infections (hepatitis B and C, cytomegalovirus, and Epstein-Barr virus), autoimmune hepatitis, primary biliary cirrhosis, sclerosing cholangitis, hemochromatosis, alpha-1-antitrypsin deficiency, Wilson's disease, history of parenteral nutrition, and use of agents known to induce steatosis (e.g., valproate, amiodarone, or vitamin E) or hepatic injury caused by substance abuse, and current consumption of more than $20 \mathrm{~g}$ of alcohol daily. None of the patients had any clinical evidence of hepatic decompensation, such as hepatic encephalopathy, ascites, variceal bleeding, or an elevated serum bilirubin level of more than twofold the upper normal limit. Other exclusion criteria were (1) tofogliflozin or glimepiride hypersensitivity or contraindications, (2) history of type 1 diabetes, (3) history of ketoacidosis, (4) history of symptoms of hypoglycemia, (5) treatment with SGLT2 inhibitor including tofogliflozin within 4 weeks of screening, (6) glinide and sulfonylurea use within 4 weeks of screening, (7) concomitant corticosteroid therapy, (8) poorly controlled unstable diabetes (ketoacidosis or an increase in $\mathrm{HbA1c}$ of more than $3 \%$ in the 12 weeks before screening), (9) poorly controlled hypertension or systolic blood pressure greater than $160 \mathrm{mmHg}$ or diastolic blood pressure greater than $100 \mathrm{mmHg}$, (10) artificial dialysis or moderate renal dysfunction, (11) poorly controlled dyslipidemia, (12) presence of a severe health problem, not being suitable for the study, (13) pregnant or breastfeeding, and (14) inability to participate in the study (including psychiatric and psychosocial problems), as assessed by the investigators.

Randomization The participants who met all the eligibility criteria and provided their written informed consent were randomly assigned on a 1:1 basis to either of the two study treatments using computer-generated randomization at Kanazawa University Hospital.

The patients in the SGLT2 inhibitor group received tofogliflozin (fixed dose of $20 \mathrm{mg} /$ day; brand name Deberza; Kowa Company Ltd., Japan) and the patients in the sulfonylurea group received glimepiride (starting from $0.5 \mathrm{mg} /$ day and titrated up to $6.0 \mathrm{mg} /$ day; Sanofi K.K., Canada) for 48 weeks.

Study Intervention The study was divided into the following four stages (Fig. 1): (1) screening, enrollment, randomization, and baseline investigations (visits 1 and 2, over a maximum period of 14 days), (2) study treatment (visits 3-5, over 48 weeks), (3) endpoint assessment, including liver biopsy and metabolic sub-studies (visit 6 for 1 week), and (4) post-treatment follow-up assessment (visit 7 for 12 weeks). If the trial investigator or the trial participant suspected an adverse event, an unscheduled visit was arranged within 2-3 days. The schedules for the study visits and data collection are summarized in Table 1 . All of the participants were asked to attend each visit under fasting states for a minimum of $8 \mathrm{~h}$ before each visit. A follow-up liver biopsy was obtained under ultrasound guidance after completion of the 48-week study treatment.

Previous treatment with oral antidiabetic drugs was continued at the same dose in participants at randomization. Glycemic control was assessed at each 12-weekly trial visit using plasma glucose and HbA1c measurements. In the event of deterioration of glycemic control, defined as $\mathrm{HbA1c}$ of greater than 9\% after the intervention, the participant will be informed

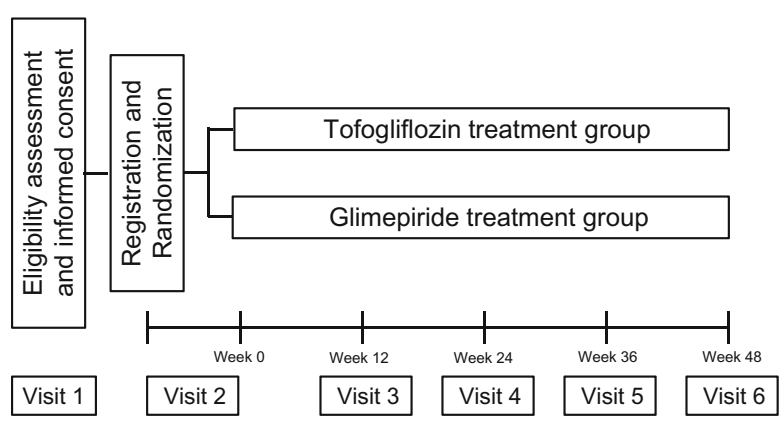

Fig. 1 Flow chart of the study schedule 
Table 1 Trial schedule of data collection

\begin{tabular}{|c|c|c|c|c|c|c|c|}
\hline & \multicolumn{2}{|l|}{ Screening } & \multicolumn{4}{|c|}{ Treatment (TD, treatment day) } & \multirow{2}{*}{$\begin{array}{l}\text { Follow-up } \\
\text { Visit } 7 \\
(12 \text { weeks } \\
\text { after } \\
\text { EOT) }\end{array}$} \\
\hline & $\begin{array}{l}\text { Visit } 1 \\
\text { (Max } \\
-12 \text { weeks } \\
\text { to TD1) }\end{array}$ & $\begin{array}{l}\text { Visit } 2 \\
(1-2 \text { weeks } \\
\text { prior to } \\
\text { TD1) }\end{array}$ & $\begin{array}{l}\text { Visit } 3 \\
\text { (Week } \\
12)\end{array}$ & $\begin{array}{l}\text { Visit } 4 \\
\text { (Week } \\
24)\end{array}$ & $\begin{array}{l}\text { Visit } 5 \\
\text { (Week } \\
36)\end{array}$ & $\begin{array}{l}\text { Visit } 6 \\
(1-7 \text { days }+ \text { week } 48 / \\
\text { EOT })\end{array}$ & \\
\hline Informed consent & $\mathrm{X}$ & & & & & & \\
\hline Clinical assessment $^{\mathrm{a}}$ & $\mathrm{X}$ & $\mathrm{X}$ & $X$ & $\mathrm{X}$ & $\mathrm{X}$ & $\mathrm{X}$ & $\mathrm{X}$ \\
\hline Vital signs ${ }^{\mathrm{b}}$ & $\mathrm{X}$ & $\mathrm{X}$ & $X$ & $\mathrm{X}$ & $\mathrm{X}$ & $\mathrm{X}$ & $\mathrm{X}$ \\
\hline Screening blood test ${ }^{c}$ & $\mathrm{X}$ & & $X$ & $\mathrm{X}$ & $\mathrm{X}$ & $\mathrm{X}$ & $X$ \\
\hline Liver biopsy & $X$ & & & & & $X$ & \\
\hline $\begin{array}{l}\text { Genes of the liver and } \\
\text { blood cells }\end{array}$ & $X$ & & & & & $\mathrm{X}$ & \\
\hline Transient elastography & $\mathrm{X}$ & & $\mathrm{X}$ & & & $\mathrm{X}$ & \\
\hline Study medication ${ }^{\mathrm{d}}$ & $\mathrm{X}$ & $\mathrm{X}$ & $\mathrm{X}$ & $\mathrm{X}$ & $\mathrm{X}$ & $\mathrm{X}$ & $\mathrm{X}$ \\
\hline Adverse/clinical events & & & $X$ & $\mathrm{X}$ & $\mathrm{X}$ & $\mathrm{X}$ & $X$ \\
\hline Bioelectrical impedance & & $\mathrm{X}$ & $\mathrm{X}$ & $\mathrm{X}$ & $\mathrm{X}$ & $\mathrm{X}$ & $\mathrm{X}$ \\
\hline $\begin{array}{l}\text { Standard blood and } \\
\text { urine test }{ }^{\mathrm{e}}\end{array}$ & & $\mathrm{X}$ & $X$ & $\mathrm{X}$ & $\mathrm{X}$ & $X$ & $\mathrm{X}$ \\
\hline $\begin{array}{l}\text { Oxidative stress } \\
\text { markers, cytokine }\end{array}$ & & $\mathrm{X}$ & & & & $X$ & \\
\hline Hepatokines & & $\mathrm{X}$ & & & & $X$ & \\
\hline $\begin{array}{l}\text { Micro RNAs and } \\
\text { exosome contents }\end{array}$ & & $\mathrm{X}$ & & & & $\mathrm{X}$ & \\
\hline $\begin{array}{l}\text { Arginine stimulation } \\
\text { test }\end{array}$ & & $\mathrm{X}$ & & & & $\mathrm{X}$ & \\
\hline $\begin{array}{l}\text { Euglycemic } \\
\text { hyperinsulinemic } \\
\text { clamp study with } \\
\text { stable isotopes }\end{array}$ & & $\mathrm{X}$ & & & & $\mathrm{X}$ & \\
\hline $\begin{array}{l}\text { Respiratory quotient } \\
\text { and basal energy } \\
\text { expenditure }\end{array}$ & & $\mathrm{X}$ & & & & $\mathrm{X}$ & \\
\hline $\begin{array}{l}\text { Proton magnetic } \\
\text { resonance } \\
\text { spectroscopy }\end{array}$ & & $\mathrm{X}$ & & & & $\mathrm{X}$ & \\
\hline $\begin{array}{l}\text { Cardiac autonomic } \\
\text { nerve activity }\end{array}$ & & $\mathrm{X}$ & & & & $\mathrm{X}$ & \\
\hline EndoPAT & & $\mathrm{X}$ & & & & $\mathrm{X}$ & \\
\hline
\end{tabular}


Table 1 continued

\begin{tabular}{|c|c|c|c|c|c|c|c|}
\hline & \multicolumn{2}{|l|}{ Screening } & \multicolumn{4}{|c|}{ Treatment (TD, treatment day) } & \multirow{2}{*}{$\begin{array}{l}\text { Follow-up } \\
\text { Visit } 7 \\
(12 \text { weeks } \\
\text { after } \\
\text { EOT) }\end{array}$} \\
\hline & $\begin{array}{l}\text { Visit } 1 \\
\text { (Max } \\
-12 \text { weeks } \\
\text { to TD1) }\end{array}$ & $\begin{array}{l}\text { Visit } 2 \\
(1-2 \text { weeks } \\
\text { prior to } \\
\text { TD1) }\end{array}$ & $\begin{array}{l}\text { Visit } 3 \\
\text { (Week } \\
12 \text { ) }\end{array}$ & $\begin{array}{l}\text { Visit } 4 \\
\text { (Week } \\
24)\end{array}$ & $\begin{array}{l}\text { Visit } 5 \\
\text { (Week } \\
36)\end{array}$ & $\begin{array}{l}\text { Visit } 6 \\
(1-7 \text { days }+ \text { week } 48 / \\
\text { EOT })\end{array}$ & \\
\hline $\begin{array}{l}\text { Treatment satisfaction } \\
\text { questionnaire }\end{array}$ & & $X$ & & & & $\mathrm{X}$ & \\
\hline Feces & & $X$ & & & & $X$ & \\
\hline $\begin{array}{l}\text { TD1 treatment day } 1, \\
\text { a Clinical assessment: } \\
\text { b Vital signs: blood pre } \\
\text { c Screening blood test: } \\
\text { d Study medication: If } \\
\text { tofogliflozin or sulfonyl } \\
\text { e Standard blood and u }\end{array}$ & $\begin{array}{l}O T \text { end of tre } \\
\text { mplete history } \\
\text { sure, heart rat } \\
\text { asting plasma } \\
\text { the study pat } \\
\text { rea } \\
\text { ine test: fill bl }\end{array}$ & $\begin{array}{l}\text { tment, EndoP } \\
\text { /examination, } \\
\text { weight, heigh } \\
\text { flucose, HbAl } \\
\text { ent meets the } \\
\text { ood count, ren }\end{array}$ & $\begin{array}{l}A T \text { periph } \\
\text { physical a } \\
\mathrm{t} \text {, waist ar } \\
\text { eligibility }\end{array}$ & $\begin{array}{l}\text { eral arteri } \\
\text { d mental } \\
\text { d hip circ } \\
\text { criteria, }\end{array}$ & $\begin{array}{l}\text { l tone te } \\
\text { condition } \\
\text { umferenc } \\
\text { e/she wi }\end{array}$ & be randomized at $\mathrm{TI}$ & to receive \\
\hline
\end{tabular}

and counseled with regard to starting open-label, long-acting insulin detemir once daily. However, the patients' participation in the trial will not be jeopardized if they do not wish to start using insulin detemir. The dose of insulin detemir has been titrated by trial investigators to ensure that the participants' standard of diabetes care will not be significantly compromised as a result of participating in our clinical trial. In addition to study medications, the participants continued to undergo lifestyle modifications (i.e., exercise, weight loss, and dietary adjustment) and management of various coexisting illnesses throughout the trial. The patients were asked to limit alcohol consumption to less than $20 \mathrm{~g} /$ day for women and $30 \mathrm{~g} /$ day for men. The participants were not allowed any new prescriptions.

All of the patients and responsible guardians received an hour of nutritional counseling by an experienced dietician before the 48-week treatment period. The experienced dieticians were unaware of the study assignments. In addition, all of the patients were given a standard calorie diet $(30 \mathrm{kcal} / \mathrm{kg}$ per day; carbohydrates, 50-60\%; fat, 20-30\%; and protein, 15-20\%) and exercise (5-6 metabolic equivalent estimations for 30 min daily) counseling before the study.

\section{Planned Outcome}

The primary outcome measure will be assessed using an intention-to-treat analysis of the proportion of evaluable patients achieving improvement in liver histology between liver biopsies at baseline and after 48 weeks of treatment. Histological improvement will be defined as improvement in histologic findings, which requires improvement by one or more points in the hepatocellular ballooning score and the fibrosis score.

Secondary endpoints include changes in (1) overall NAFLD Activity Score, (2) liver enzymes, (3) body composition, (4) fasting plasma glucose levels and glucose metabolism assessed with an arginine stimulation test, (5) organspecific insulin sensitivity, (6) lipid profiles, (7) renal function and electrolyte balances, (8) oxidative stress (urinary 8-isoprostane and urinary 8-hydroxydeoxyguanosine [8-OHdG]), (9) cytokines [tumor necrosis factor (TNF)-alpha, leptin, and adiponectin] levels, (10) hepatokines (selenoprotein P and LECT-2) levels, (11) 
organ-specific fat accumulation, (12) oxidative and non-oxidative glucose disposal, (13) energy expenditure, (14) respiratory quotients, (15) autonomic nerve function, (16) minerals and bone metabolism, (17) endothelial functions, (18) fatty acid profiles, (19) gut microbiota profiling in feces, (20) comprehensive gene expression profiles in blood cells and liver, (21) micro RNAs and exosome contents, and (22) treatment satisfaction. Factors associated with the changes in these parameters will be investigated.

\section{Data Collection}

\section{Liver Histopathology}

A single pathologist, who was blinded to the clinical information and order in which the biopsies were obtained, analyzed all of the biopsies twice and at separate times. The sections were cut from a paraffin block and stained with hematoxylin-eosin and silver reticulin stains. The biopsied tissues were scored for steatosis (0-3), stage (1-4), and grade (1-3) as previously described [10], according to the standard criteria for grading and staging [11]. The NAFLD Activity Score was calculated as the unweighted sum of the scores for steatosis (0-3), lobular inflammation (0-3), and ballooning (0-2) [12].

Clinical and Laboratory Data Type 2 diabetes was considered present if the fasting plasma glucose was at least $7 \mathrm{mmol} / \mathrm{L}$ and if the 2 -h 75-g oral glucose tolerance test plasma glucose was at least $11.1 \mathrm{mmol} / \mathrm{L}$ or if the patients had a recorded diagnosis in their medical records.

Clinical information, including age, sex, medication, and body measurements, was obtained for each patient. Body measurements of height, body weight, heart rate, systolic/diastolic blood pressure, and waist circumferences were recorded. Body composition, such as body fat mass and fat-free mass, was determined using a bioelectrical impedance analysis (Tanita BC-118D; Tanita, Tokyo, Japan). Venous blood samples, which were obtained after the patients had fasted overnight, were used to evaluate blood chemistry. Blood samples were analyzed for full blood count, liver enzymes (serum aspartate aminotransferase, serum alanine aminotransferase, and plasma gamma-glutamyltransferase), renal function (blood urea nitrogen levels, creatinine, and estimate glomerular filtration rate), electrolyte balances, lipid profile (total cholesterol, high-density lipoprotein, and triglycerides), cytokines (TNFalpha, leptin, and adiponectin), HbA1c, and plasma glucose. Serum fatty acids were measured using a gas chromatograph (Shimizu GC 17A; Kyoto, Japan) at SRL Inc. (Tokyo, Japan). Oxidative stress markers, such as urinary 8-isoprostane and 8-OHdG, were measured (SRL Inc.). A fecal sample was collected and stored at $-80^{\circ} \mathrm{C}$ [13]. The liver gene expression was collected, stored, and measured as described previously [14].

Arginine Stimulation Test The arginine stimulation test has been demonstrated to be a valid method for evaluating residual $\beta$-cell function even during periods of hyperglycemia [15]. Arginine (30 g) was administered intravenously by infusing a 10\% L-arginine hydrochloride solution over $30 \mathrm{~min}$. Blood samples (plasma glucose, C-peptide immunoreactivity [CPR], and glucagon) were collected at seven time points $(0,15,30,45,60,90$, and $120 \mathrm{~min})$. Circulating CPR and glucagon were used to construct an arginine stimulated time-response curve. The values of the area under the concentration-time curve for CPR and glucagon between time 0 and $120 \mathrm{~min}$ were calculated using the trapezoidal rule. The value of arginine $\triangle \mathrm{CPR}$ was defined as the difference between the maximal and basal levels of CPR during the arginine test [15].

Liver Stiffness Liver stiffness was evaluated using transient elastography (Fibroscan, Echosens, France). The median value and interquartile range (IQR) of ten validated measurements were recorded within the range of $2.5-75 \mathrm{kPa}$ [16]. The XL probe was used on individuals who had a body mass index (BMI) greater than $30 \mathrm{~kg} / \mathrm{m}^{2}$ or when the Fibroscan 502 Touch machine recommended its use over the M-probe. 
Organ-Specific Insulin Sensitivity Organspecific insulin sensitivity was estimated by the hyperinsulinemic euglycemic clamp study using an artificial pancreas (model STG-55; Nikkiso, Tokyo, Japan) combined with the stable isotope-labeled $\left[6,6-{ }^{2} \mathrm{H}_{2}\right]$ glucose infusion. We calculated and defined organ-specific insulin resistance in the liver, skeletal muscle, and adipose tissue as described previously [17]. Hepatic insulin resistance indices were calculated as the product of fasting hepatic glucose production (HGP) and fasting plasma insulin concentration and suppression of HGP by insulin during a clamp study. The skeletal muscle insulin resistance index was calculated as insulin-stimulated glucose disposal (Rd) [18].

Energy Metabolism Whole-body indirect calorimetry was performed during the hyperinsulinemic euglycemic clamp study using an AE$310 \mathrm{~s}$ Aeromonitor (Minato Medical Science Company, Osaka, Japan). The respiratory quotient was defined as the ratio of carbon dioxide production $\left(\mathrm{VCO}_{2}\right)$ to whole-body oxygen consumption $\left(\mathrm{VO}_{2}\right)$. Basal energy expenditure was calculated from $\mathrm{VO}_{2}$ and $\mathrm{VCO}_{2}$ using the Weir equation. We calculated glucose oxidation from the respiratory gas exchange and nitrogen excretion in urine [19]. Non-oxidative glucose disposal was estimated by subtracting the glucose oxidation rate from $\mathrm{Rd}$ [20].

Organ-Specific Fat Accumulation Intrahepatic lipid (IHL) and intramyocellular lipid (IMCL) were measured as previously reported $[18,21]$. IHL of the liver's right lobe and IMCL of the soleus muscle were measured using ${ }^{1} \mathrm{H}$ magnetic resonance spectroscopy (MRS) and a whole-body 3.0 T MR system (Signa HDxt 3.0 T; General Electric Healthcare, Milwaukee, WI, USA). Voxels $\left(3.06 \times 3.06 \times 3.0 \mathrm{~cm}^{3}\right.$ for liver and $2.06 \times 2.06 \times 2.0 \mathrm{~cm}^{3}$ for soleus muscle) were positioned in the liver or soleus muscle to avoid blood vessels and visible interfacial fat, and the voxel sites were carefully matched at each examination, and the MR spectral raw data were processed to quantify IHL and IMCL using the LCModel software (Version 6.3-0C; Stephen Provencher, Oakville, ON, Canada) [18].
Cardiac Autonomic Nerve Activity The participants underwent 24-h ambulatory Holter electrocardiograms. The results were transformed into the frequency-domain waveforms that included high-frequency (HF) power $(0.15-0.40 \mathrm{~Hz})$ and low-frequency (LF) power (0.04-0.15 Hz). LF power, HF power, and LF/HF ratio represent sympathetic nerve activity, parasympathetic nerve activity, and sympathovagal balance, respectively [22].

Endothelial Function Peripheral endothelial function is measured using an EndoPAT-2000 device (Itamar Medical, Caesarea, Israel). A peripheral arterial tonometry probe was attached to the fingers of both hands of the subject. The reactive hyperemia index (RHI) was determined according to the manufacturer's instructions. Endothelial dysfunction is indicated as RHI less than 1.67 [23].

Treatment Satisfaction Treatment satisfaction was assessed using the Diabetes Treatment Satisfaction Questionnaire (DTSQ) at baseline and at the end of the study as a secondary outcome. The overall treatment satisfaction score was calculated as the sum of DTSQ items 1 (Q1, satisfaction with the treatment), 4 (Q4, convenience of the treatment), 5 (Q5, flexibility of the treatment), 6 (Q6, understanding of your diabetes), 7 (Q7, recommend to others), and 8 (Q8, wish to continue treatment). Items 2 (Q2, perceived hyperglycemia frequency) and 3 (Q3, perceived hypoglycemia frequency) were treated as separate variables.

Case Report Forms The case report forms (CRFs) include baseline/follow-up medical histories and physical examinations to capture comorbidities and concomitant medications in the trial's electronic database. Other CRFs incorporated in the electronic database include the following: laboratory data including thyroid function and questionnaire results; safety monitoring during the treatment follow-up periods; histopathology reports of liver biopsy specimens; noninvasive markers of liver disease; and adverse event reporting and study drug dispensing forms for study treatment adherence and accountability. Treatment compliance, 
including the evidence of dosage and clinical events, is to be monitored at each study visit.

\section{Data Analysis}

\section{Sample Size Estimation}

At the time of the study design, we had no available data to estimate the histological response with a 48-week treatment using tofogliflozin and glimepiride. Therefore, we estimated the sample size on the basis of findings of other studies on non-SGLT2 inhibitors as follows: on the basis of clinical trials on nonSGLT2 inhibitors for NAFLD that had improvements in liver histology as a primary endpoint, we assumed that $52.6-69.0 \%$ of patients undergoing treatment would demonstrate an improvement in NAFLD $[14,24]$. We estimated that in $17.6-19.0 \%$ of the placebo control arm patients, there would be an improvement in liver histology on the basis of the literature $[14,24]$. We calculated the sample size as 14 in each group, with a significance level of 0.05 (type I error) and a power of 0.90 (type II error). This design required 40 evaluable patients in the treatment group. The published literature in NAFLD trials reported on average a participant withdrawal rate of $10-20 \%[14,25]$. Therefore, we randomized our recruitment target of 40 patients in a 1:1 allocation ratio to either tofogliflozin or glimepiride.

\section{Statistical Analysis}

Data are expressed as the mean \pm 2 standard deviations. SPSS software, version 22.0 (SPSS Inc., Chicago, Illinois, USA), is being used for all the statistical analyses. For univariate comparisons between patient groups, a Student's $t$ test or Mann-Whitney $U$ test was used, as appropriate, followed by Bonferroni's multiple comparison test. A $P$ value less than 0.05 was considered to be statistically significant.

\section{DISCUSSION}

This study will result in the first report on the efficacy of treatment with an SGLT2 inhibitor or sulfonylurea on liver pathology in patients with
NAFLD and type 2 diabetes in a 48-week openlabel randomized trial. We will compare the efficacy of the SGLT2 inhibitor and sulfonylurea in ameliorating liver histology in NAFLD. The enrollment of the required sample size will be completed in September 2020 and the final results are expected by the end of 2021 .

The efficient recruitment of patients with NAFLD for clinical trials remains a challenge when it requires a liver biopsy. This situation with respect to liver biopsy has several limitations, including sampling heterogeneity, invasive nature, and patient reluctance, especially in repeated sampling. Nevertheless, liver biopsy will be required for trials in NAFLD until the accuracy of serial measurements of noninvasive markers is formally validated. In addition, liver biopsy still remains a gold standard for evaluating not only fibrosis but also steatosis, inflammation, and hepatocyte damage in NAFLD.

In the present study, we tested our hypothesis that lowering glucose and increasing insulin reduce liver fibrosis in NAFLD. The SGLT2 inhibitor lowers insulin levels and the sulfonylurea increases insulin levels, while both lower glucose levels. Therefore, by comparing the effects of these hypoglycemic agents and by extracting factors associated with alteration in liver histology, we aimed to clarify whether a decrease in glucose, increase in insulin, or weight reduction contributes to reducing liver histological scores. Through these findings, we may establish the disease entity of "diabetic steatohepatitis" in the pathology of NAFLD.

SGLT2 inhibitors may reduce body weight and ectopic fat accumulation. However, it still remains unclear whether these inhibitors reduce whole-body insulin resistance and which organ is responsible for altered insulin sensitivity. Our initial hypothesis regarding liver fat is that SGLT2 inhibitors sense liver glycogen deficiency, stimulate sympathetic activity, enhance lipolysis to generate glycerol, upregulate hepatic gluconeogenesis, and thereby reduce liver fat. The secondary efficacy endpoints in the present study include organspecific insulin sensitivity evaluated with the hyperinsulinemic euglycemic clamp study combined with stable isotope-labeled glucose 
infusion, insulin/glucagon secretion evaluated by the arginine stimulation test, ectopic fat accumulation evaluated by ${ }^{1} \mathrm{H}$ MRS and bioelectrical impedance analysis, sympathetic nerve activity estimated from the heart rate variability by using Holter electrocardiograms, comprehensive gene expression analyses in the liver and blood cells, and gut microbiota profiling. Using these surrogate markers, we may clarify the mechanisms underlying the SGLT2 inhibitor/sulfonylurea-mediated alteration in body weight and whole-body energy metabolism.

\section{ACKNOWLEDGEMENTS}

Funding. This work was supported, in part, by Grants-in-Aid from the Ministry of Education, Culture, Sports, Science 19K08975 (Y.T. 40507042) and by research grants from Kowa Company Ltd. The journal's Rapid Service fee was paid by Grants-in-Aid from the Ministry of Education, Culture, Sports, Science 19K08975.

Editorial Assistance. We received assistance from Enago ${ }^{\mathrm{TM}}$ English Editing Service (Crimson Interactive Pvt. Ltd.), which was paid from Grants-in-Aid from the Ministry of Education, Culture, Sports, Science 19K08975.

Authorship. All authors met the International Committee of Medical Journal Editors criteria for authorship for this study, take responsibility for the integrity of this work, and have given their approval for this version to be published.

Disclosures. Toshinari Takamura received lecture fees and received research grants from Kowa Company Ltd. Yumie Takeshita received lecture fees from Kowa Company Ltd. Takehiro Kanamori, Takeo Tanaka, Yuka Kaikoi, Yuki Kita, Noboru Takata, Noriho Iida, Kuniaki Arai, Tatsuya Yamashita, Kenichi Harada, Toshifumi Gabata, Hiroyuki Nakamura, and Shuichi Kaneko have nothing to disclose.
Compliance with Ethics Guidelines. The institutional review board of each participating institution approved the study protocol in compliance with the Declaration of Helsinki and current legal regulations in Japan. All procedures followed were in accordance with the ethical standards of the responsible committee on human experimentation (institutional and national) and with the Helsinki Declaration of 1964, as revised in 2013. Informed consent was obtained from all patients that are willing to participate in the study.

Open Access. This article is distributed under the terms of the Creative Commons Attribution-NonCommercial 4.0 International License (http://creativecommons.org/licenses/ by-nc/4.0/), which permits any noncommercial use, distribution, and reproduction in any medium, provided you give appropriate credit to the original author(s) and the source, provide a link to the Creative Commons license, and indicate if changes were made.

\section{REFERENCES}

1. Hamaguchi E, Takamura T, Sakurai M, et al. Histological course of nonalcoholic fatty liver disease in Japanese patients: tight glycemic control, rather than weight reduction, ameliorates liver fibrosis. Diabetes Care. 2010. https://doi.org/10.2337/dc090148 .

2. Davies MJ, D'Alessio DA, Fradkin J, et al. Correction to: management of hyperglycaemia in type 2 diabetes, 2018. A consensus report by the American Diabetes Association (ADA) and the European Association for the Study of Diabetes (EASD). Diabetologia. 2019;62:873. https://doi.org/10.1007/ s00125-019-4845-x.

3. Bolinder J, Ljunggren Ö, Kullberg J, et al. Effects of dapagliflozin on body weight, total fat mass, and regional adipose tissue distribution in patients with type 2 diabetes mellitus with inadequate glycemic control on metformin. J Clin Endocrinol Metab. 2012;97:1020-31. https://doi.org/10.1210/jc.20112260.

4. Hayashizaki-Someya Y, Kurosaki E, Takasu T, et al. Ipragliflozin, an SGLT2 inhibitor, exhibits a prophylactic effect on hepatic steatosis and fibrosis induced by choline-deficient L-amino acid-defined 
diet in rats. Eur J Pharmacol. 2015;754:19-24. https://doi.org/10.1016/j.ejphar.2015.02.009.

5. Honda Y, Imajo K, Kato T, et al. The selective SGLT2 inhibitor ipragliflozin has a therapeutic effect on nonalcoholic steatohepatitis in mice. PLoS One. 2016;11:1-13. https://doi.org/10.1371/journal. pone.0146337.

6. Obata A, Kubota N, Kubota T, et al. Tofogliflozin improves insulin resistance in skeletal muscle and accelerates lipolysis in adipose tissue in male mice. Endocrinology. 2016;157:1029-42. https://doi.org/ 10.1210/en.2015-1588.

7. Kuchay MS, Krishan S, Mishra SK, et al. Effect of empagliflozin on liver fat in patients with type 2 diabetes and nonalcoholic fatty liver disease: a randomized controlled trial (E-LIFT Trial). Diabetes Care. 2018;41:1801-8. https://doi.org/10.2337/ dc18-0165.

8. Sumida Y, Murotani K, Saito M, et al. Effect of luseogliflozin on hepatic fat content in type 2 diabetes patients with non-alcoholic fatty liver disease: a prospective, single-arm trial (LEAD trial). Hepatol Res. 2019;49:64-71. https://doi.org/10.1111/hepr. 13236.

9. UK Prospective Diabetes Study (UKPDS) Group. Intensive blood-glucose control with sulphonylureas or insulin compared with conventional treatment and risk of complications in patients with type 2 diabetes (UKPDS 33). Lancet. 1998;352: 837-53. http://www.ncbi.nlm.nih.gov/pubmed/ 9742976

10. Sakurai M, Takamura T, Ota T, et al. Liver steatosis, but not fibrosis, is associated with insulin resistance in nonalcoholic fatty liver disease. J Gastroenterol. 2007;42:312-7. https://doi.org/10.1007/s00535006-1948-

11. Brunt EM, Janney CG, Di Bisceglie AM, et al. Nonalcoholic steatohepatitis: a proposal for grading and staging the histological lesions. Am J Gastroenterol. 1999;94:2467-74. https://doi.org/10.1111/j.15720241.1999.01377.x.

12. Kleiner DE, Brunt EM, Van Natta M, et al. Design and validation of a histological scoring system for nonalcoholic fatty liver disease. Hepatology. 2005;41:1313-21. https://doi.org/10.1002/hep. 20701.

13. Iida N, Mizukoshi E, Yamashita T, et al. Overuse of antianaerobic drug is associated with poor postchemotherapy prognosis of patients with hepatocellular carcinoma. Int J cancer. 2019. https:// doi.org/10.1002/ijc.32339.
14. Takeshita $\mathrm{Y}$, Takamura $\mathrm{T}$, Honda $\mathrm{M}$, et al. The effects of ezetimibe on non-alcoholic fatty liver disease and glucose metabolism: a randomised controlled trial. Diabetologia. 2014. https://doi.org/ 10.1007/s00125-013-3149-9.

15. Tsuchiyama $\mathrm{N}$, Takamura $\mathrm{T}$, Ando $\mathrm{H}$, et al. Possible role of alpha-cell insulin resistance in exaggerated glucagon responses to arginine in type 2 diabetes. Diabetes Care. 2007;30:2583-7. https://doi.org/10. 2337/dc07-0066.

16. Pang JXQ, Zimmer S, Niu S, et al. Liver stiffness by transient elastography predicts liver-related complications and mortality in patients with chronic liver disease. PLoS One. 2014;9:e95776. https://doi. org/10.1371/journal.pone.0095776.

17. Cobelli C, Mari A, Ferrannini E. Non-steady state: error analysis of Steele's model and developments for glucose kinetics. Am J Physiol. 1987;252: E679-89. https://doi.org/10.1152/ajpendo.1987. 252.5.E679.

18. Kato K, Takamura T, Takeshita Y, et al. Ectopic fat accumulation and distant organ-specific insulin resistance in Japanese people with nonalcoholic fatty liver disease. PLoS One. 2014;9:e92170. https://doi.org/10.1371/journal.pone.0092170.

19. Frayn KN. Calculation of substrate oxidation rates in vivo from gaseous exchange. J Appl Physiol. 1983;55:628-34. https://doi.org/10.1152/jappl. 1983.55.2.628.

20. Yokoyama H, Mori K, Emoto M, et al. Non-oxidative glucose disposal is reduced in type 2 diabetes, but can be restored by aerobic exercise. Diabetes Obes Metab. 2008;10:400-7. https://doi.org/10. 1111/j.1463-1326.2007.00716.x.

21. Ryysy L, Häkkinen AM, Goto T, et al. Hepatic fat content and insulin action on free fatty acids and glucose metabolism rather than insulin absorption are associated with insulin requirements during insulin therapy in type 2 diabetic patients. Diabetes. 2000;49:749-58. https://doi.org/10.2337/ diabetes.49.5.749.

22. Perini R, Veicsteinas A. Heart rate variability and autonomic activity at rest and during exercise in various physiological conditions. Eur J Appl Physiol. 2003;90:317-25. https://doi.org/10.1007/ s00421-003-0953-9.

23. Bonetti PO, Pumper GM, Higano ST, et al. Noninvasive identification of patients with early coronary atherosclerosis by assessment of digital reactive hyperemia. J Am Coll Cardiol. 2004;44:2137-41. https://doi.org/10.1016/j.jacc.2004.08.062. 
24. Sanyal AJ, Chalasani N, Kowdley KV, et al. Pioglitazone, vitamin $\mathrm{E}$, or placebo for nonalcoholic steatohepatitis. N Engl J Med. 2010;362:1675-85. https://doi.org/10.1056/nejmoa0907929. pioglitazone.
25. Ratziu V, Charlotte F, Bernhardt C, et al. Long-term efficacy of rosiglitazone in nonalcoholic steatohepatitis: results of the Fatty Liver Improvement by Rosiglitazone Therapy (FLIRT 2) extension trial. Hepatology. 2010;51:445-53. https://doi.org/10.1002/hep.23270. 